\title{
Implementing Direct Electron Detection Camera K2 and Dose Fractionation for Near Atomic Resolution Single Particle CryoEM
}

\author{
Xueming $\mathrm{Li}^{1}$, Shawn Zheng ${ }^{2}$, David A. Agard ${ }^{1,2}$ and Yifan Cheng ${ }^{1}$ \\ 1. The W.M. Keck Advanced Microscopy Laboratory, Department of Biochemistry and Biophysics, \\ University of California San Francisco, 600 16th Street, San Francisco, CA 94158, USA. \\ 2. The Howard Hughes Medical Institute, University of California San Francisco, 600 16th Street, San \\ Francisco, CA 94158, USA
}

As a versatile tool in structural biology, single particle electron cryomicroscopy (cryoEM) has achieved milestones of determining near atomic resolution 3D reconstruction of large protein assemblies, such as icosahedral viruses, without the need of crystal. However, applying this technique routinely to a broad range of biological molecules is still extremely challenge. One of the bottlenecks is the image-recording medium. Either photographic film or traditional CCD camera are not sufficient for efficient acquisition of high-resolution cryoEM data. Another one is electron beam induced image motion that blurs highresolution information and deteriorates image quality. Beam-induced motion is unpredictable and thus it is very difficult to be eliminated [1].

The newly developed direct electron detection cameras has proven to be superior than both photographic film and CCD camera in recording high-resolution images of frozen hydrated protein sample embedded in a thin layer of vitreous ice [2, 3 and 4]. We have characterized a K2 Summit ${ }^{\mathrm{TM}}$ (Gatan Inc., USA) and implemented it for collecting high-resolution cryoEM data for near atomic resolution structure determination by single particle cryoEM. The detective quantum efficiency (DQE) of K2 Summit is significantly higher, at both high and low frequency ranges, than both scintillator based CCD camera and photographic films. Furthermore, K2 Summit has an internal frame rate of 400 frame/second that is sufficient, under the appropriate imaging condition, to count individual primary electron events in a practical manner. The ability to count and centroid primary electron event to sub-pixel area make the K2 Summit a true digital camera without readout noise. The high output frame rate also enables recording image with a novel "movie" or "dose fractionation" mode that offers great potential to facilitate near atomic resolution single particle cryoEM.

We have implemented the new K2 Summit camera into our single particle cryoEM data acquisition routine (Figure 1). Achieving an optimal performance of the K2 camera requires a relative low electron dose rate on camera to minimize coincidence loss caused by overcrowding of electron events. Our recent work suggests collecting image at a dose rate of $\sim 10 \mathrm{e}^{-} / \mathrm{pixel} / \mathrm{sec}$ or less on camera [4]. With such a low dose rate on camera, the exposure time needed to record an image with a required total electron dose on specimen, such as $30 \mathrm{e}^{-} / \AA^{2} / \mathrm{sec}$, would be around 10 seconds or more, depending on the magnification at which an image is taken. Thus, potential stage instability together with beam-induced image motion become major factors that deteriorating otherwise superb images. Under influence of both beam-induced motion and stage instability, only less than 1\% images are "perfect" one suitable for near atomic resolution 3D reconstruction. We thus developed a novel algorithm to correct for image motion by accurately measure the displacements between all subframes within one "dose fractionated" image stack. Our test has shown that motion correction improved the quality of almost every image to the level that is suitable for near-atomic resolution $3 \mathrm{D}$ reconstruction. 
We have now implemented this algorithm into a graphic processing unit (GPU) based program for fast processing of dose fractionated image stacks. We also developed a semi-automated image acquisition program, UCSFImage, to facilitate streamlined acquisition of dose fractionated image stacks of frozen hydrated biological molecules following strict well-established low-dose data acquisition procedure. UCSFImage also coordinate on-the-fly motion correction for immediate feedback. Using this system, we have collected a full dataset of dose-fractionated image stacks of archaeal $20 \mathrm{~S}$ proteasome and determined its $3 \mathrm{D}$ reconstruction to $3.3 \AA$ resolution.

\section{References:}

[1] RM Glaeser et al, Ultramicroscopy 111 (2007) p. 90.

[2] MG Campbell et al, Structure 20 (2012) p. 1823.

[3] BE Bammes, et al, Journal of Structural Biology 177 (2012) p. 589.

[4] X Li et al, Nature Methods (2013) Submitted.

[5] The authors acknowledge funding from the National Science Foundation (NSF grant DBI-0960271), HHMI and NIH (R01GM098672, YC).

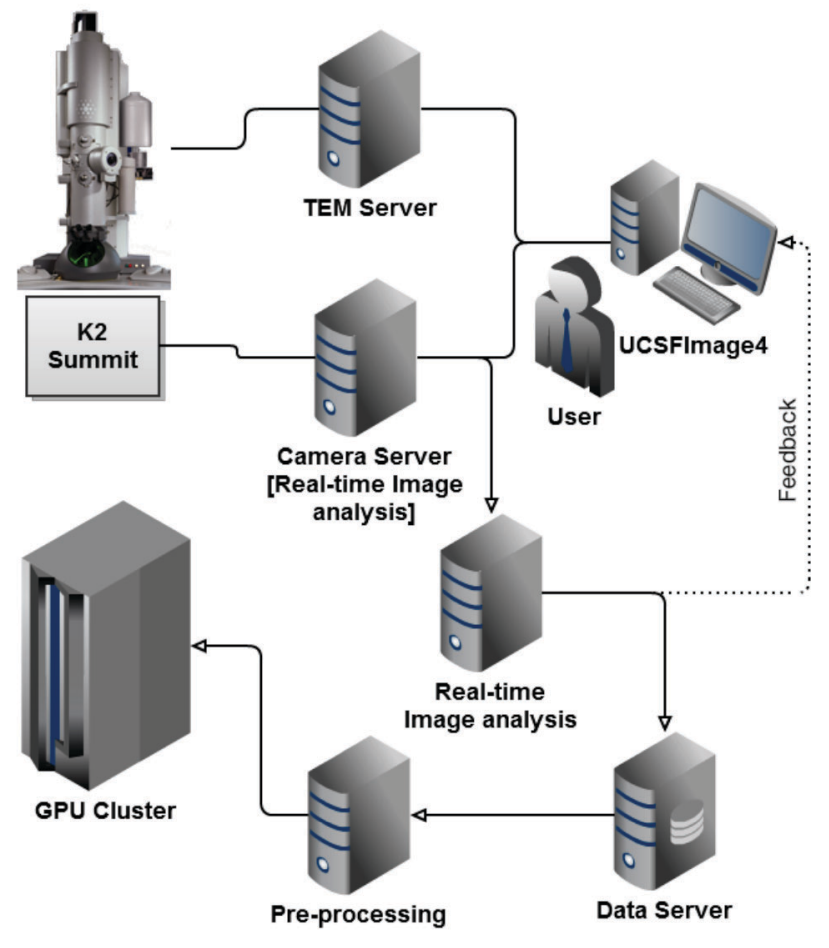

Figure 1. Implementing K2 Summit into a streamlined semi-automated image acquisition procedure. A streamlined data acquisition through the K2 camera and on-the-fly motion correction is coordinated by the UCSFImage4. On-the-fly motion correction is carried out on GPU based computing. 